\title{
Spinal Myoclonus - A Rare Complication of Spinal Anaesthesia
}

\author{
M S Prabhu ${ }^{1}$
}

\section{Abstract}

Spinal segmental myoclonus is a rare type of myoclonic disorder that may occur during spinal anaesthesia. A few cases of spinal myoclonus have been reported after administration of intrathecal bupivacaine. The exact cause and pathophysiology of spinal myoclonus is unknown. We report a case of 28 -year-old female who underwent caesarean section under spinal anaesthesia with $0.5 \%$ hyperbaric bupivacaine. Myoclonic movements appeared in the early postoperative period in the left lower limb. The myoclonus was acute and transient. The patient recovered completely without any neurological complications. After ruling out all the possible causes, spinal myoclonus diagnosis was made, and the patient was discharged on the $3^{\text {rd }}$ postoperative day.

Keywords: Spinal anaesthesia, Spinal myoclonus, Myoclonus

\section{Introduction}

Spinal myoclonus indicates the distribution of muscle jerking limited to muscles innervated by one or two contagious spinal segments $[1,2]$. There are several possible causes of spinal myoclonus including AV malformation, spinal cord tumor, trauma, multiple sclerosis, amyotropic lateral sclerosis, paraneoplastic syndromes and viral infections. Spinal myoclonus has been reported following intrathecal administration of local anaesthetics, opioids, radiocontrast agents and intrathecal catheter placement, though the incidence is relatively uncommon. We report a case of spinal myoclonus in a patient following hyperbaric bupivacaine spinal anaesthesia for elective caesarean section.

\section{Case Report}

A 28-year-old second gravida with 38 weeks of gestation was posted for elective caesarean section. She had an uneventful spinal anaesthesia two years ago for the first caesarean section. She had no significant medical history, allergy, or previous neurological disease. Her routine preoperative blood investigations were within normal limits. A written anaesthesia consent was obtained, and she was kept nil by mouth for 6 hours before the surgery.

In the operation theatre, intravenous line patency was checked, and ringer lactate fluid was started. The usual monitoring was used (ECG, non-invasive blood pressure and pulse oximetry). Spinal anaesthesia with 25-gauge Quincke needle was performed at L3-L4 interspace with the patient in the seated position. $2.2 \mathrm{ml}$ of $0.5 \%$ hyperbaric bupivacaine was injected through the needle after freely flowing cerebrospinal fluid was obtained. A live, term, female baby weighing $2.3 \mathrm{~kg}$ was born and the surgery got over uneventfully in approximately 45 minutes. Patient was shifted to room after 30 minutes of observation in the recovery room. The anaesthesia team was subsequently called to the ward to review the patient about 2 hrs. after spinal anaesthesia because she developed involuntary jerky movements in the left lower limb. These myoclonic movements were sudden, short bursts of muscle contractions in the left leg, approximately 10 episodes, occurring every 3-5 minutes and each lasting 5-10 seconds. There were no such movements in the right leg or upper extremities. The patient was conscious and alert throughout the episodes. The patient could move her toes but did not feel pin prick or cold sensation up to $\mathrm{L} 1$ dermatome, indicating incomplete recovery from the spinal block. Reassurance was given to the patient and injection midazolam was kept ready in case the myoclonus recovers. The blood sugar was within normal limits and serum electrolytes was sent and found to be within normal limits. A neurology consult was obtained. EEG was done, and no abnormality detected. Patient was followed up for the next 2 days and was no 
other such episodes, hence she was discharged on the $3^{\text {rd }}$ postoperative day.

\section{Discussion}

Myoclonus is a movement disorder characterized by sudden, brief shock like jerks. Based on the source of its generation, it can be classified into cortical, subcortical, spinal or peripheral myoclonus.

Cortical myoclonus usually affects the distal upper limbs and face because of the largest cortical representation of these body areas. These jerks are stimulus sensitive especially to touch and typically occurs on voluntary action. Subcortical myoclonus (brain stem myoclonus) is manifested by generalized jerks, involvement of proximal to distal muscles and bilateral representation. These are also stimulus sensitive like cortical myoclonus. Both cortical and subcortical myoclonus can be ruled out in our case because of the exclusive single lower limb representation, stimulus insensitive and occurrence during rest. Spinal segmental myoclonus confined to one or few contiguous myotomes and may occur irregularly with the frequency as low as 1-2 per minute or as high as $100-200$ per minute. It is generally resistant to supraspinal influences such as sleep or voluntaryaction.

Psychogenic myoclonus is a rare form that may occur following an external trauma or spontaneously. These jerks are distractible and have exaggerated stimulus sensitivity, may be focal or generalised. It is difficult to distinguish from organic myoclonus.

On approaching a patient with myoclonus, nature of onset and precipitating factors are important. Acute onset is usually seen in hepatic and renal failure, thyrotoxicosis, electrolyte and metabolic imbalances (hypoglycemia, hyponatremia, hypokalemia), some neuro infectious diseases. Chronic progression is a characteristic of neurodegenerative disorders. In our patient, it was of acute onset in the immediate postoperative period and all the laboratory investigations were within normal limits.

Electrophysiology includes EEG, EMG is useful to detect the origin of myoclonus. In cortical myoclonus EEG spikes precedes the myoclonus. On contrary, in subcortical there is no EEG changes, but distribution and mode of spread of myoclonus recording from EMG differentiates it from spinal myoclonus. In psychogenic myoclonus, EMG bursts will be always more than $50 \mathrm{~ms}$, because it is unlikely to produce voluntary EMG spikes less than that and also in EEG trigger spikes occurs just prior to a myoclonic jerks. In our case EEG was normal, hence cortical involvement ruled out. We did not do EMG, because, there was no recurrence of event and clinical distribution and characteristics ruled out subcortical and psychogenic myoclonus.

There are a few cases of myoclonus reported in the literature following spinal anaesthesia. Fox et al. [5], Yamashita et al. [6], Binita et al. [7], Alfa and Bamgbade [4], all reported myoclonic jerks following local anaesthetic injection in subarachnoid space, which got settled down in a period of time either with benzodiazepine or anticonvulsant. The suggested pathophysiology of spinal myoclonus includes abnormal hyperactivity of local anterior horn neurons, aberrant local axon re excitations, loss of inhibitory function of local dorsal horn interneurons and loss of inhibition from suprasegmental descending pathways $[3,4]$.

In our case intrathecal bupivacaine appears to be the most likely cause, because there was no history of neurological disorder, a normal neurological examination and a normal EEG and blood investigations. The local anaesthetic may have induced spinal cord irritation resulting in spontaneous and repetitive discharges of the anterior horn cell groups. Some have suggested that the effect of bupivacaine on inhibitory neurons may have led to a loss of inhibitory function in the spinal cord and heightened irritability of the alpha motor neuron leading to myoclonus [8]. Usually, this type of myoclonus resolves after the disappearance of the drug effects. However, in some cases specific treatment including benzodiazepines and anticonvulsants is needed.

\section{Conclusion}

Spinal myoclonus is very rare phenomenon to occur as a result of spinal anaesthesia with bupivacaine, which can be effectively managed with reassurance and benzodiazepines after ruling out other neurological abnormalities. 
Declaration of patient consent: The authors certify that they have obtained all appropriate patient consent forms. In the form, the patient has given his consent for his images and other clinical information to be reported in the Journal. The patient understands that his name and initials will not be published, and due efforts will be made to conceal his identity, but anonymity cannot be guaranteed.

\section{Conflict of interest: Nil Source of support: None}

\section{References}

1. Caviness JN, Brown P. Myoclonus: current concepts and recent advances. Lancet Neurol. 2004; 3:598-607.

2. Cassim F, Houdayer E. Neurophysiology of myoclonus. Neurophysiol Clin 2006; 36 (5-6):281-91.

3. Friedreich N. Neuropathologische Beobach-tungen I. Paramyoklonus Multiplex, Virchows Archiv für pathologische Anatomie und Physiologie und für klinische Medicin 1881; 86: 421-30.

4. Alfa JA, Bamgbade OA. Acute myoclonus following spinal anaesthesia. EurJ Anaesthesiol 2008; 25:256-7.

5. Fox EJ, Villanueva R, Schutta HS. Myoclonus following spinal anesthesia. Neurology 1979; 29:379-80.
6. Yamashita A, Matsumoto M, Matsumoto S, Itoh M, Kawai K, Sakabe T. A comparison of neurotoxic effects on the spinal cord of tetracaine, lidocaine, bupivacaine, and ropivacaine administered intrathecally in rabbits. Anesthesia and Analgesia 2003; 97: 512-9.

7. Panigrahi B, Samaddar DP, Mahapatra BC, Varghese K. Spinal Myoclonus Following Bupivacaine Spinal Anaesthesia for Varicose Vein Stripping. Indian Journal of Anaesthesia 2008;52 (3):331-333.

8. Zamidei L, Bandini M, Michelagnoli G, Campostrini R, Consales G. Propriospinal myoclonus following intrathecal bupivacaine in hip surgery: a case report. Minerva Anestesiol. 2010; 76:290-293.

\section{How to Cite this Article}

Prabhu MS | Spinal Myoclonus - A Rare Complication of Spinal Anaesthesia | Journal of Anaesthesia and Critical Care Case Reports | September-December 2021; 7(3): 17-

19. 